\title{
Acute Glucose Fluctuations and Chronic Sustained Hyperglycemia as Risk Factors for Cardiovascular Diseases in Patients with Type 2 Diabetes
}

Author

Affiliation
C. Colette ${ }^{1}$, L. Monnier ${ }^{2}$

${ }^{1}$ Laboratory of Human Nutrition, University Institute of Clinical Research, Montpellier cedex 5, France ${ }^{2}$ Department of Metabolic Diseases, Lapeyronie Hospital, Montpellier cedex 5, France
Key words

- Type 2 diabetes

- chronic hyperglycemia

- acute glucose fluctuations

received 27.02.2007

accepted 25.04.2007

Bibliography

DOI 10.1055/s-2007-985157

Horm Metab Res 2007;

39: 683-686

(c) Georg Thieme Verlag KG

Stuttgart · New York

ISSN 0018-5043

Correspondence

Prof. L. Monnier

Department of Metabolic

Diseases

Lapeyronie Hospital

34295 Montpellier cedex 5

France

Tel.: + 33/467/338402

Fax: + 33/467/339591

I-monnier@chu-montpellier.fr

\section{Abstract}

$\nabla$

Chronic hyperglycemia, usually assessed from HbA1c determinations, results in excessive glycation and generation of oxidative stress. As a consequence, chronic hyperglycemia has been identified as a risk factor for diabetes complications leading to accelerated atherosclerosis. Both fasting and postprandial hyperglycemia contribute to this process. However the acute glucose fluctuations that occur in diabetes have been recently described as an additional factor

\section{Introduction}

$\nabla$

To day, nobody can deny the role of diabetes in the development of specific microvascular complications and the high incidence of accelerated atherosclerosis [1]. Although a large number of studies have investigated and compared the roles of the many factors involved in diabetic vascular complications, an accurate assessment of their respective contributions is still difficult. However, as demonstrated by many trials, microvascular and macrovascular complications are mainly $[2,3]$ or partly dependent on "dysglycemia", which has two components: chronic sustained hyperglycemia (including fasting and chronic postprandial hyperglycemia) and acute glycemic fluctuations from peaks to nadirs. Both components lead to diabetic complications through two main mechanisms: excessive protein glycation and activation of oxidative stress.

\section{The Brownlee theory or the theory of oxidative stress $\nabla$}

The two mechanisms which we have just evoked were unified in an elegant theory that suggested that the glycemic disorders observed in diabetic that activates the oxidative stress. As a consequence, acute glucose swings, including upward (postprandial) and downward (interprandial) fluctuations can be considered as risk factors for cardiovascular events and should be included in the "dysglycemia" of diabetes in combination with fasting and postprandial hyperglycemia. As postprandial glucose is a contributor of both acute glucose fluctuations and chronic sustained hyperglycemia, it remains difficult to know whether these 2 mechanisms are equivalent or not equivalent risk factors for cardiovascular disease.

patients result in an activation of oxidative stress with an overproduction of superoxide by the mitochondrial electron-transfer chain [4] (๑ Fig. 1). This activation in turn produces a cascade of such deleterious metabolic events as enhanced polyol activity, increased formation of advanced glycation end-products, activation of protein kinase $C$ and nuclear factor $\kappa \mathrm{B}$, and increased hexosamine pathway flux [4]. It is now well established that hyperglycemia both at fasting and during postprandial periods results in exaggerated and accelerated glycation. For instance, all the studies conducted in type 1 and type 2 diabetes have clearly shown a strong positive relationship between HbA1c levels and plasma glucose levels at fasting and over postprandial periods $[5,6]$, the strongest correlation being observed between HbA1c and mean plasma glucose levels [7]. The latter relationship was considered sufficiently demonstrative to serve as a reference in the recent Standards of Medical Care in Diabetes that are published every year by the American Diabetes Association [8]. To day, HbA1c is unanimously recognized as a reliable marker for the overall glucose exposure and its direct consequence, an excessive rate of glycation $[9,10]$. The simplicity of this concept masks more complex phenomena because $\mathrm{HbA1c}$ is an integrator of both fasting 


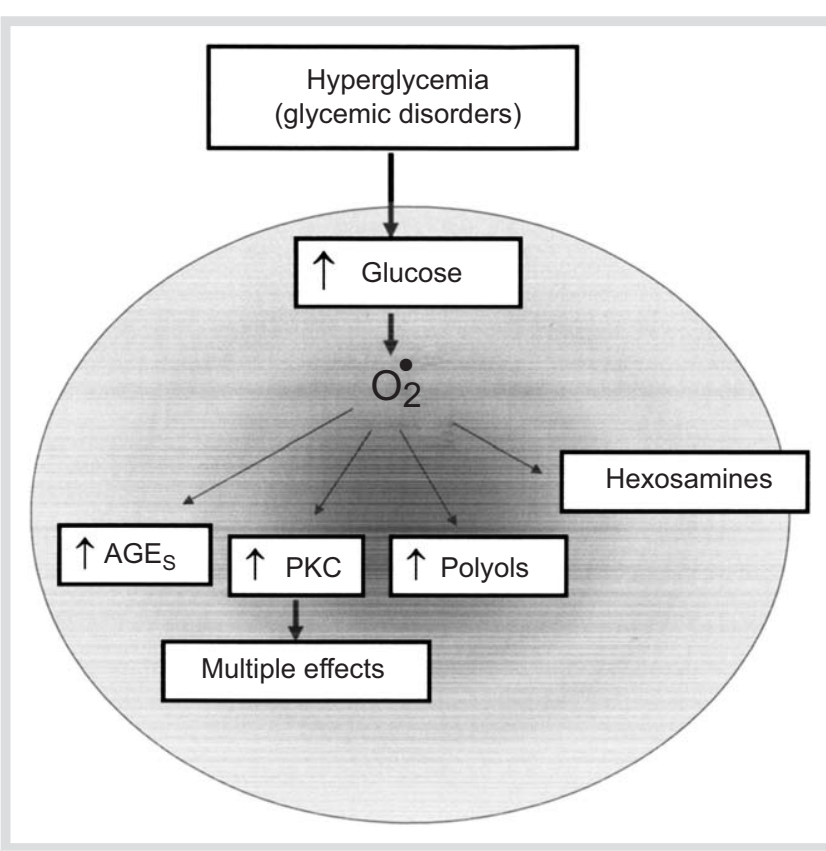

Fig. 1 Metabolic alterations activated by hyperglycemia in endothelial cells. Theory of oxidative stress for vascular damages in diabetes [4]. $\mathrm{O}_{2}$ : Superoxide anion AGEs: advanced glycation end products PKC: Protein kinase $C$.

and postprandial glycemic disorders. As a consequence, it is not surprising that either fasting or postprandial hyperglycemia were identified separately or concomitantly as major risk factors for diabetic complications. The UKPDS study pointed out HbA1c and fasting blood glucose levels as major predictors of diabetesrelated complications. This study demonstrated that the risks for myocardial infarction and microvascular complications were diminished by 14 and $37 \%$, respectively, for each $1 \%$ reduction in HbA1c [3]. On the other hand, in the Diabetes Intervention Study published in 1996 by Hanefeld [11], postprandial hyperglycemia was a better predictor of subsequent myocardial infarction and mortality than fasting hyperglycemia. These results, confirmed by other studies, suggest that postprandial hyperglycemia is an independent risk factor for macrovascular diseases [12,13]. However, risk factors are not limited to fasting and postprandial hyperglycemia. Other factors such as dyslipidemia, hypertension, and hemostasis dysfunction (all are at least partly associated with the insulin resistance of type 2 diabetes) can be involved in macrovascular complications. Furthermore, even though we consider only glycemia alterations, fasting and postprandial hyperglycemia may be not the only components of diabetic complications. Another risk factor is probably the glucose variability within a day, especially the acute glucose fluctuations. This raises the following question: Are chronic hyperglycemia and acute glucose fluctuations equivalent risk factors for cardiovascular disease? In order to respond to this question, the first part of this review article is mainly devoted to the analysis of the contributions of the two components of the "dysglycemia": sustained chronic hyperglycemia and acute glucose fluctuations to the activation of oxidative stress and thus to the respective impacts of these glycemic disorders on diabetic complications. Several markers have been used to assess oxidative stress and the antioxidant status in patients with diabetes. The short plasma half-life of these markers is one of the limiting factors for the assessment of oxidative stress in plasma samples.
Thus, when available, urinary determinations provide a more reliable estimation of the activation of oxidative stress than plasma measurements $[14,15]$. Accordingly, the determination of such specific isoprostane isomers as the 8-iso-PGF $2 \alpha$ in urine has been proposed. Isoprostanes are collectively formed from free radical-mediated oxidation of arachidonic acid [16]. As this fatty acid is ubiquitously distributed in cell membranes, measurements of urinary isoprostanes most likely provide an excellent reflection of the activation of oxidative stress in the whole body. In the different studies that have been conducted in diabetes, plasma and urinary metabolites have been alternatively or simultaneously used as oxidative stress markers.

As free radical production has been reported to be increased in patients with diabetes mellitus, it has been suggested that hyperglycemia may directly contribute to the generation of oxidative stress. There is cogent evidence from several studies that hyperglycemia is associated with an increased formation and urinary excretion rate of 8 -iso-PGF $2 \alpha$ [17]. The urinary excretion rate of 8 -iso-PGF $2 \alpha$ was found to be significantly increased in type 2 diabetic patients as compared with age-matched healthy subjects. Furthermore, significant correlations were observed between blood glucose and urinary 8 -iso-PGF2 $\alpha$ suggesting that the activation of oxidative stress may be, at least in part, related to the determinants of diabetic control. We have recently confirmed these results by showing that the mean urinary excretion rates of 8 -iso-PGF $2 \alpha$ were significantly higher $(\mathrm{p}<0.01)$ in patients with type 2 diabetes (mean $\pm \mathrm{SD}=482 \pm 206 \mathrm{pg} / \mathrm{mg}$ of creatinine) than in nondiabetic healthy subjects $(275 \pm 85 \mathrm{pg} / \mathrm{mg}$ of creatinine) [18]. Improved metabolic control has been shown to be associated with a significant reduction in urinary excretion rates of both 8 -iso-PGF2 $\alpha$ and 11-dehydrothromboxane (TXM) by 32 and $48 \%$, respectively, in 21 patients with type 2 diabetes. Vitamin E supplementation led to complete normalization of 8 -iso-PGF2 $\alpha$ excretion rates [19]. Moreover, changes in 8 -iso-PGF2 $\alpha$ excretion were accompanied by similar reduction in TXM excretion, consistent with a cause-and-effect relation between enhanced lipid peroxidation and persistent platelet activation. Increase of plasma 8 -iso-PGF $2 \alpha$ is an early event in the evolution of type 2 diabetes and could precede the development of endothelial dysfunction.

\section{Contribution of acute glucose fluctuations to the activation of oxidative stress \\ $\nabla$}

Postprandial glucose results in both acute and sustained hyperglycemia since postprandial periods have a 4-hour duration. Several interventional or epidemiological studies suggest that acute hyperglycemia after a meal or glucose load may be an independent predictor of risk for vascular event in type 2 diabetes [20]. Such a relationship results from an increased generation of reactive oxygen species during acute hyperglycemia, leading to acute oxidative damage to the vascular endothelium. For instance, acute hyperglycemia after a glucose load in type 2 diabetes is associated with an acute increase in plasma concentrations of 8 -iso-PGF2 $\alpha$. The role of postprandial hyperglycemia in the generation of oxidative stress was particularly investigated by Ceriello et al., who demonstrated that the production of free radicals was increased during the postprandial period [21] and that this increment was proportional to the magnitude of the postprandial glucose excursions. For instance, fasting nitrotyrosine, a metabolite derived from nitrosamine stress, was 


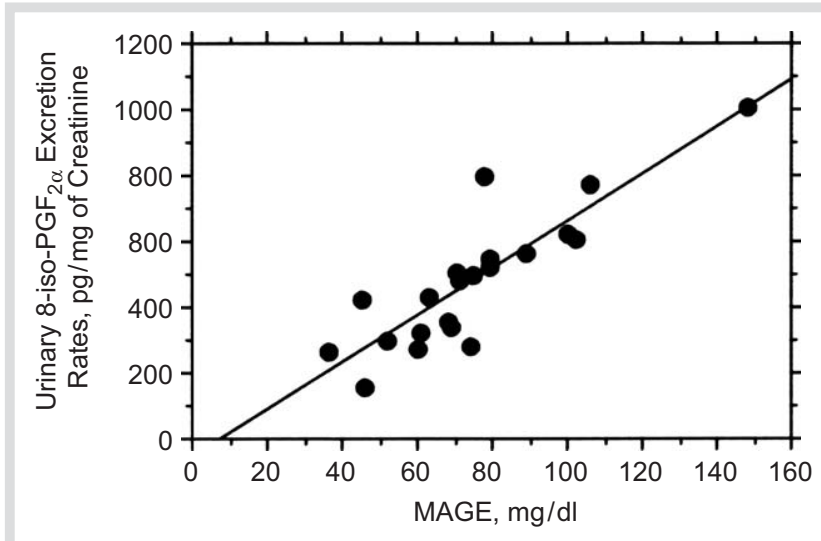

Fig. 2 Linear correlation between 24-hour urinary excretion rates of 8-iso-prostaglandin F2 $\alpha$ and Mean Amplitude of Glycemic Excursions (MAGE) in 21 patients with type 2 diabetes (from [18], printed with permission of JAMA).

significantly increased in the diabetic patients. An additional increase was observed during postmeal periods. Reduction of the postmeal glucose excursions by using a premeal bolus of rapid insulin analog (Aspart) resulted in parallel decrements in glycemic and nitrotyrosine responses [22]. This provides direct evidence for a link between acute rather than chronic hyperglycemia and free radical damage in diabetes.

Although postprandial glucose is usually the major contributor of glucose variability, other fluctuations (especially downward fluctuations) must be taken into account. In a recent study [18], we have demonstrated that the urinary excretion rate of 8-isoPGF $2 \alpha$ was highly and positively correlated with the glycemic variability assessed from the mean amplitude of glycemic excursions (MAGE). For this purpose, the patients' glucose profiles were obtained over 48 hours from continuous glucose monitoring system (CGMS) data. The calculation of the MAGE was made by measuring the arithmetic mean of the difference between consecutive peaks and nadirs, provided that the difference was greater than the standard deviation (SD) around the mean glucose values. The relationship is indicated in 0 Fig. $2(r=0.86$, $\mathrm{p}<0.001$ ). A statistically significant correlation was also observed with the mean postprandial glucose incremental area under the curve (AUCpp) but the relationship was less significant $(p=0.009)$. It thus appears that the triggering effect of acute glycemic excursions on oxidative stress should be integrated into glycemic disorders that are much broader than acute postmeal spikes. As a consequence, the concept that postprandial "hyperglycemic spikes" are "dangerous waves", should be extended to both upward (postprandial) and downward (interprandial) acute fluctuations of glucose around a mean value. This observation may provide an explanation for some of the epidemiological observations of the DCCT. For instance, in the subgroups with a sustained HbA1c of $9 \%$ for the entire study duration, the risk of retinopathy was reduced by more than $50 \%$ in the intensive control group compared with the conventional group, even though these two subgroups of patients had the same HbA1c. The difference might have been due to a lower intraday glucose variability in the intensive control group. However, this hypothesis was not confirmed by a recent analysis of the data sets collected in the DCCT. In this retrospective study, Kilpatrick et al. [23] reported that the mean blood glucose, i.e., sustained chronic hyperglycemia, was predictive of microvascular complications in patients

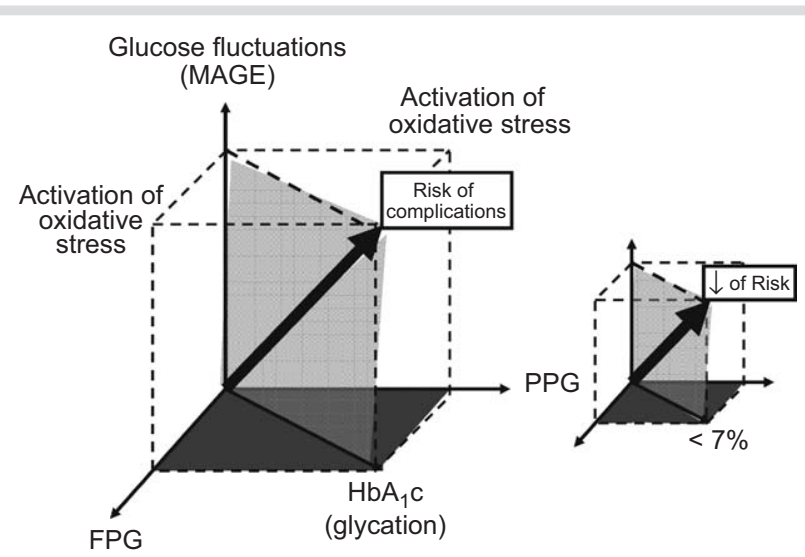

Fig. 3 Model suggested for illustrating the pathophysiological impacts of excess glycation and activation of oxidative stress on the risk of diabetic complications (diagonal solid arrow). The contributions of the three components of dysglycemia, i.e., hyperglycemia at fasting (FPG), hyperglycemia during postprandial periods (PPG), and acute glucose fluctuations (MAGE) are indicated on the $x, y$, and $z$ axis, respectively.

with type 1 diabetes while within-day glucose variability was not. It should be noted, however, that in this study the instability of blood glucose was calculated as the standard deviation (SD) around the mean of a seven-point glycemic profile measured at each patient's quarterly visit. With such a methodology, the authors probably selected not major fluctuations but rather a composite of both major and minor swings with a majority of minor ones. Furthermore, Kilpatrick et al. [23] probably blunted the contributions of major glucose fluctuations because there are many reasons to think that the four pre(inter)- prandial and the three postprandial glucose values included in the sevenpoint profile did not perfectly coincide with the glucose nadirs and peaks, respectively. The following example should be useful to explain the superiority of the MAGE index for assessing glucose variability compared with the SD of a seven-point glucose profile. Consider two patients with type 2 diabetes who have similar HbA1c and SD of glucose fluctuations around the mean. Assume that one subject has many minor glucose fluctuations and one or two major swings per day, while the other patient exhibits moderate glucose fluctuations over 24 hours. Despite similar SD of glucose around the mean, these two patients should exhibit very different MAGE values and thus Kilpatrick's use of SD as a definitive measure of glucose variability is questionable. Even though the MAGE determination requires continuous glucose monitoring, our opinion is that this index should be the "gold standard" for assessing glucose fluctuations in all prospective interventional trials designed to estimate glucose variability. Therefore, expanded use of continuous glucose sensors would certainly be useful for conducting such trials.

In conclusion, the pathophysiology of diabetic complications can be considered as the result of two major deleterious metabolic alterations (excessive glycation and generation of oxidative stress) that are activated by three main glycemic disorders: hyperglycemia both at fasting and during postprandial periods and acute glucose fluctuations ( $\bullet$ Fig. 3). At present, there is no doubt that excessive levels of glucose at fasting and during postprandial periods activate the glycation process, which can be investigated as a whole by measuring the HbA1c levels. In addition to hyperglycemia at fasting, acute or sustained hyperglycemia over postprandial periods and more generally acute glucose 
fluctuations around the mean glucose value activate oxidative stress. The resulting effect is the risk of complications depicted by the diagonal arrow of a geometric cube whose three-dimensional coordinates on the three axes are FPG, PPG, and glucose fluctuations. According to this model, a global antidiabetic therapeutic strategy should be aimed at reducing the values of the three coordinates, i.e., the volume of the cube, and therefore the magnitude of the diagonal arrow that illustrates the risk for diabetic complications ( $\bullet$ Fig. 3). However, several questions remain to be solved. For instance and at present, it is not possible to know whether the famous Aristotle's aphorism can be applied to the dysglycemia of patients with type 2 diabetes or not: "The whole, i.e., the dysglycemia and its consequence - the risk of complication - is greater than the sum of its parts (FPG, PPG, and acute glucose fluctuations)".

\section{References}

1 Laakso L, Lehto S. Epidemiology of macrovascular disease in diabetes. Diabetes Rev 1997; 5: 294-315

2 The Diabetes Control and Complications Trial Research Group. The effect of intensive treatment of diabetes on the development and progression of long-term complications in insulin-dependent diabetes mellitus. N Engl J Med 1993; 329: 977-986

3 Stratton IM, Adler AI, Neil HAW, Matthews DR, Manley SE, Cull CA, Hadden D, Turner RC, Holman RR, the UK Prospective Diabetes Study Group. Association of glycaemia with macrovascular and microvascular complications of type 2 diabetes (UKPDS 35): prospective observational study. BMJ 2000; 321: 405-412

4 Brownlee M. Biochemistry and molecular cell biology of diabetic complications. Nature 2001; 414: 813-820

5 Avignon A, Radauceanu A, Monnier L. Nonfasting plasma glucose is a better marker of diabetic control than fasting plasma glucose in type 2 diabetes. Diabetes Care 1997; 20: 1822-1826

6 El-Kebbi IM, Ziemer DC, Cook CB, Gallina DL, Barnes CS, Philipps LS, Utility of casual postprandial glucose levels in type 2 diabetes management. Diabetes Care 2004; 27: 335-339

7 Rohlfing CL, Wiedmeyer HS, Little RR, England JD, Tennill A, Goldstein DE. Defining the relationship between plasma glucose and HbA1c. Diabetes Care 2002; 25: 275-278

8 American Diabetes Association. Standards of medical care in diabetes2007. Diabetes Care 2007; 30 (Suppl 1): S4-S41

9 Sacks DB, Bruns DE, Goldstein DE, Mac Laren NK, Mac Donald JM, Parrott $M$. Guidelines and recommendations for laboratory analysis in the diagnosis and management of diabetes mellitus (position statement). Diabetes Care 2002; 25: 750-786
10 Gorus F, Mathieu C, Gerlo E. How should HbA1c measurements be reported? Diabetologia 2006; 49: 7-10

11 Hanefeld M, Fischer S, Julius U, Schulze J, Schwanebeck U, Schmechel H, Ziegelasch HJ, Lindner J. Risk factors for myocardial infarction and death in newly detected NIDDM: the Diabetes Interventional Study, 11-year follow-up. Diabetologia 1996; 39: 1577-1583

12 Ceriello A, Hanefeld M, Leiter L, Monnier L, Moses A, Owens D, Tajima N, Tuomilehto J. Postprandial glucose regulation and diabetic complications. Arch Int Med 2004; 164: 2090-2095

13 Cavalot F, Petrelli A, Traversa M, Bonomo K, Fiora E, Conti M, Anfossi G, Costa G, Trovati $M$. Postprandial blood glucose is a stronger predictor of cardiovascular events than fasting blood glucose in type 2 diabetes mellitus, particularly in women: lessons from the San Luigi Gonzaga Diabetes Study. J Clin Endocrinol Metab 2006; 91: 813-819

14 Morrow JD, Hill KE, Burk RF, Nammour TM, Badr KF, Roberts LJ. A series of prostaglandin F2-like compounds are produced in vivo in humans by non-cyclooxygenase free radical-catalyzed mechanism. Proc Natl Acad Sci USA 1991; 87: 9383-9387

15 Roberts LJ, Morrow JD. Measurement of F(2)-isoprostanes as an index of oxidative stress in vivo. Free Radic Bio Med 2000; 28: 505-513

16 Basu S. Metabolism of 8-iso-prostaglandin F2 $\alpha$. FEBS Lett 1998; 428 : 32-36

17 Davi G, Falco A, Patrono C. Lipid peroxidation in diabetes mellitus. Antioxid Redox Signal 2005; 7: 256-258

18 Monnier L, Mas E, Ginet C, Michel F, Villon L, Cristol J-P, Colette C. Activation of oxidative stress by acute glucose fluctuations compared with sustained chronic hyperglycemia in patients with type 2 diabetes. JAMA 2006; 295: 1681-1687

19 Davi G, Ciabattoni G, Consoli A, Mezzetti A, Falco A, Santarone S, Pennese E, Vitacolonna E, Bucciarelli T, Cotantini F, Capani F, Patrono C. In vivo formation of 8-Iso-Prostaglandin F2 $\alpha$ and platelet activation in diabetes mellitus. Effects of improved metabolic control and vitamin $\mathrm{E}$ supplementation. Circulation 1999; 99: 224-229

20 Sampson MJ, Gopaul N, Davies IR, Hughes DA, Carrier MJ. Plasma F2 Isoprostanes. Direct evidence of increased free radical damage during acute hyperglycemia in type 2 diabetes. Diabetes Care 2002; 25: 537-541

21 Ceriello A. Postprandial hyperglycemia and diabetes complications. Is it time to treat? Diabetes 2005; 54: 1-7

22 Ceriello A, Quagliaro L, Catone B, Pascon R, Piazzola M, Bais B, Marra G, Tonutti L, Taboga C, Motz E. Role of hyperglycemia in nitrotyrosine postprandial generation. Diabetes Care 2002; 25: 1439-1443

23 Kilpatrick ES, Rigby AS, Atkin SL. The effect of glucose variability on the risk of microvascular complications in type 1 diabetes. Diabetes Care 2006; 29: 1486-1490 